九州大学学術情報リポジトリ

Kyushu University Institutional Repository

\title{
Characteristics of Vibration Transmissibility for Corrugated Paperboard
}

Park, Jongmin

Department of Bioindustrial Machinery Engineering, Pusan National University

Kim, Ghiseok

Department of Mechanical Engineering, Michigan State University

Kim, Hakj in

School of Biosystems \& Biomaterials Science and Engineering, Seoul National University

Kwon, Soongoo

Department of Bioindustrial Machinery Engineering, Pusan National University

他

https://doi.org/10.5109/20328

出版情報 : 九州大学大学院農学研究院紀要. 56 (2)，pp. 327-333，2011-09. 九州大学大学院農学研究院 バージョン：

権利関係 : 


\title{
Characteristics of Vibration Transmissibility for Corrugated Paperboard
}

\author{
Jongmin PARK ${ }^{1}$, Ghiseok KIM ${ }^{2}$, Hakjin KIM ${ }^{3}$, Soongoo KWON ${ }^{1}$, \\ Muneshi MITUSOKA ${ }^{4 *}$, Eiji INOUE $^{4}$ and Takashi OKAYASU ${ }^{4}$
}

\author{
(Received April 28, 2011 and accepted May 9, 2011)
}

\begin{abstract}
Corrugated fiberboard are typically lightweight and inexpensive with high stiffness-to-weight and strength-to-weight ratios as an environmental-friendly packaging materials. In order to optimize the application of corrugated paperboard for packaging cushioning materials, it is essential that both a vibration transmissibility test and drop shock test be completed for the transmissibility-frequency curve and dynamic cushion curve respectively. This paper deals with the characterization of properties of corrugated paperboard relevant to its application for protective packaging in transportation, such as vibration transmissibility, resonant frequency, damping ratio, and maximum dynamic stress.

The main feature of the paper is the evaluation on the vibration transmissibility of corrugated paperboard with corrugation shape and the equilibrium atmospheric conditions by a sinusoidal sweep vibration test. Additionally, damping ratio and maximum dynamic stress were evaluated by linear vibration theory. All the works provide basic data relevant to its application for protective packaging in transportation.
\end{abstract}

Keywords: corrugated paperboard, vibration transmissibility, paper-based cushioning material, resonant frequency, damping ratio

\section{INTRODUCTION}

A cushion system is a protective media that provides an interface between a fragile product and potentially harmful environment such as shock and vibration. An impact on a product occurs mostly during material handling as a probability function, whereas vibration always exists during transportation. All cushioning materials experience increasing or decreasing acceleration at specific frequencies. By using these features, a cushion packaging design that reduces the products transmitted acceleration under critical acceleration can be achieved.

Both corrugated paperboard and honeycomb paperboard are environmental-friendly packaging cushioning materials with a sandwich structure. These materials are mainly used as structural members of transport packaging design and they are an alternative to conventional plastic foam cushions for environmental preservation. The necessary components for a structural member include compressive resistance, shock absorbing characteristics and vibration transmissibility. Because of inadequate information for these paper-based cushioning materials in Korea, an optimal cushion packaging design was not achieved with considering features of products and packaging materials. The design remained at a conventional level and resulted in over-packaging or underpackaging.

For corrugated containers, the compressive resist-

${ }^{1}$ Department of Bioindustrial Machinery Engineering, Pusan National University, Korea

${ }^{2}$ Department of Mechanical Engineering, Michigan State University, East Lansing, MI, USA

${ }^{3}$ School of Biosystems \& Biomaterials Science and Engineering, Seoul National University, Korea

${ }^{4}$ Department of Bioproduction Environmental Science, Graduate School of Kyushu University, Japan

* Corresponding author (E-mail: mitsuoka@bpes.kyushu-u. ac.jp) ance, buckling behavior and creep properties were studied by Biancolini and Brutti (2003)2 ${ }^{2}$, Bronkhorst and Riedemann (1994) ${ }^{3}$, Mckee et al. (1963). ${ }^{10}$ For corrugated paperboards, the compression strength, crush strength, bending defection and flexural stiffness were investigated by Hahn et al. (1992) $)^{8}$, Lee et al. (2004) $)^{9}$, Urbanik et al. (2001). ${ }^{13}$

Guo et al. (2008) analyzed the characterization of creep properties and recoverability of double-wall corrugated paperboard at different combined conditions of relative humidity and compression loads. ${ }^{5}$ Wang (2008) researched the relations between cushioning behavior of honeycomb paperboard and height, relative density and basis weight and showed relative density of paper honeycomb core has a significant effect on the cushioning properties of honeycomb paperboards; however, the basis weight of the liner has only little effect. ${ }^{14}$ Guo and Zhang (2004) evaluated dynamic cushion curve of honeycomb paperboard by a series of experimental studies on the drop shock machine and analyzed the resonance and vibration transmissibility of honeycomb paperboards by the vibration tests with sinusoidal sweep and found two or three resonances. Accordingly, honeycomb paperboards can greatly diminish the vibration at high frequency region because vibration transmissibility at resonance frequencies that exist over $350 \mathrm{~Hz}$ is insignificant. ${ }^{4}$ Sek and Kirkpatrick (1997) predicted the cushioning properties of corrugated fiberboard from static and quasidynamic compression data. ${ }^{12}$

Shock and vibration characteristics of cushioning materials could be varied by repeated shocks during transportations. Especially, paper-based cushioning materials such as corrugated paperboard can be significantly affected than foamed plastic cushioning materials by environmental shock and relative humidity during transportations. It is crucial to analyze the transmissibility of corrugated paperboard according to the corrugation shape and relative humidity because of the com- 
plexity and difficulty in the quantitative assessment of shock and vibration characteristics.

When a corrugated paperboard is used as a cushioning material, it is a prerequisite to analyze a damping ratio, resonant frequency and amplifying band that includes resonant frequency for static loading because resonance should be excluded. Moreover, moisture absorption of corrugated paperboard affects the variation of vibration transmissibility according to equilibrium atmosphere and represents complicated vibration behavior according to corrugation shape. Currently, it is difficult to find the research about these features of corrugated paperboard.

In this study, we researched the maximum vibration transmissibility, resonant frequency, damping ratio and maximum dynamic stress according to equilibrium atmosphere condition and corrugation shape.

\section{MATERIALS AND METHODS}

\section{Equipment design}

Equipment for vibration transmissibility of corrugated paperboard was comprised of electromagnetic shaker (LS-100, LING Electronics Inc., USA), 4-channel A/D board embedded controller to control the vibration profile, PZT accelerometer for vibration control (Kistler, range: $100 \mathrm{G}$, sensitivity: $50.44 \mathrm{mV} / \mathrm{G}$, frequency response: $1 \sim 25 \mathrm{kHz}$ ), light-weight PZT accelerometer with flexible

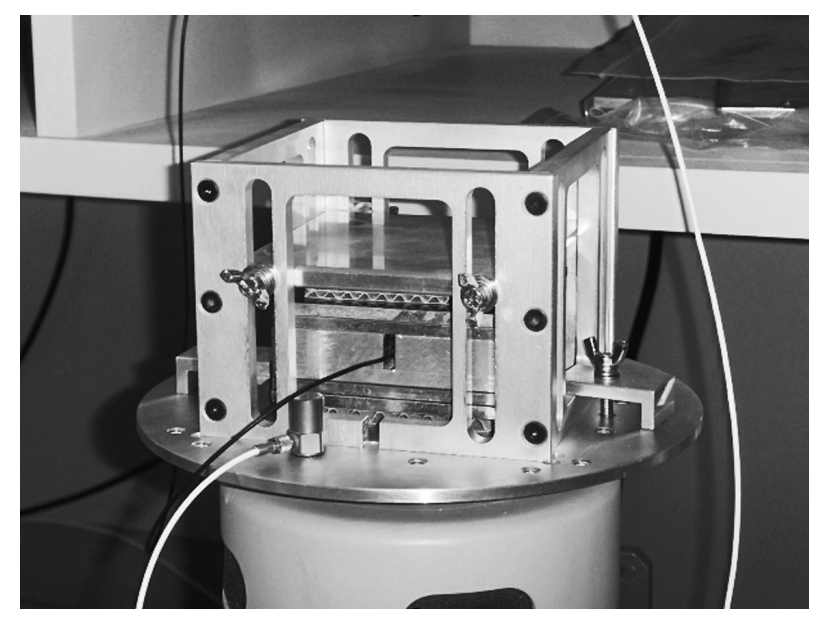

Fig. 1. Vibration test set-up. cable for vibration signal measurement (Kistler, range: $500 \mathrm{G}$, sensitivity: $9.80 \mathrm{mV} / \mathrm{G}$, frequency response: $1 \sim 25 \mathrm{kHz}$ ) and sample fixture for fixing the corrugated paperboard sample on shaker table.

We put great emphasis on the design of sample fixture shown in Figs. 1 and 2. It was designed to apply the vibration force into samples with fixed on shaker table. Because of the rigidity and light-weight features of fixture, aluminium material was used with CNC precision machining.

Weight of block can be added with 5 steps. In order to accurately analyze the vibration transmissibility feature of corrugated paperboard, the resonant frequency of sample fixture itself that include blocks should not be overlapped. Based on the results of vibration response for sample fixture within frequency range and static weight that determined by several pretests, any resonance was not appeared within test frequency range in Fig. 3.

\section{Materials}

DW(double-wall) and SW(single-wall) corrugated paperboards shown in table 1 were used in study. In order to compare the vibration transmissibility qualitatively according to corrugation shape and equilibrium atmosphere conditions, corrugated paperboard consisted of both K180 as all corrugating medium and liner composed with analogous paper was adapted.

Dimension of specimen is $70 \times 70 \mathrm{~mm}$ and equilibrium atmospheric conditions were $23^{\circ} \mathrm{C}-\mathrm{rh} 50 \%, 23^{\circ} \mathrm{C}-$ rh $70 \%$ and $23^{\circ} \mathrm{C}-$ rh $90 \%$ respectively.

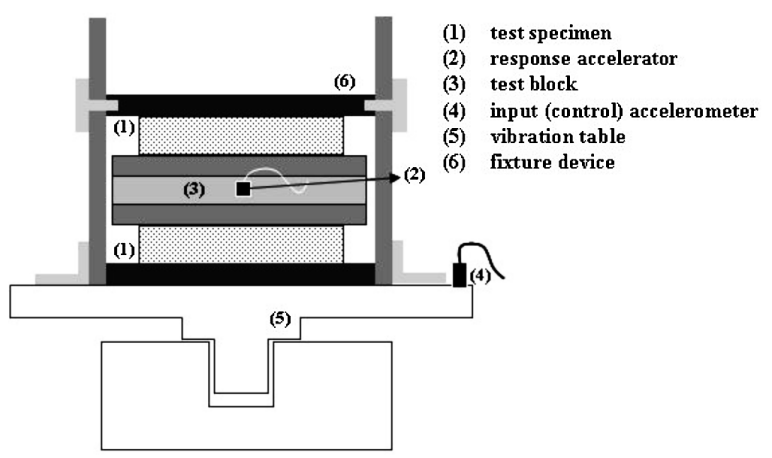

Fig. 2. Schematic diagram showing fixture device of test specimen and accelerometer placement.

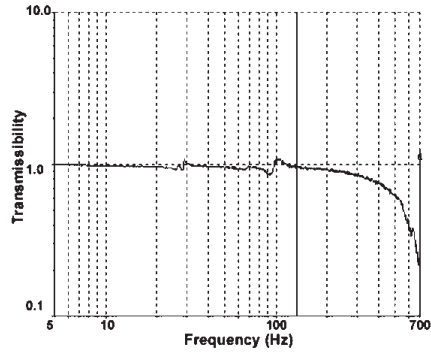

(a) $3.94 \mathrm{~N}$

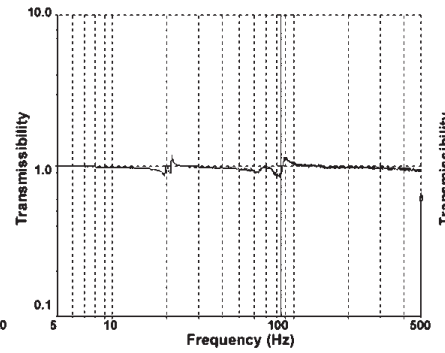

(b) $15.76 \mathrm{~N}$

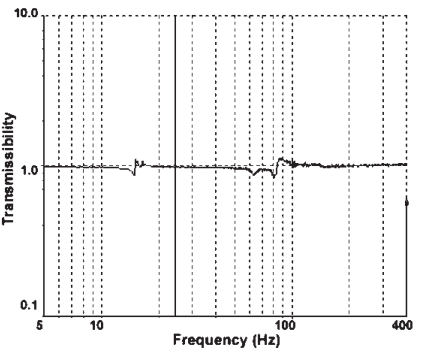

(c) $27.63 \mathrm{~N}$

Fig. 3. Vibration response of sample fixture device for the range of test frequency in varying static loading. 
Table 1. Corrugated paperboards used for the vibration test

\begin{tabular}{|c|c|c|c|}
\hline Flute & & Board combination $^{1)}$ & Physical characteristics \\
\hline $\mathrm{A} / \mathrm{F}$ & 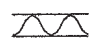 & $\mathrm{KA} 180^{2)} / \mathrm{K} 180 / \mathrm{K} 180^{3)}$ & \multirow{4}{*}{$\begin{array}{l}\text { Height(mm), wave length(mm), and Take- } \\
\text { up-factor of flute : (A/F) 4.6, 8.8, and 1.6; } \\
\text { (B/F)2.6, 6.0, and 1.4 } \\
\text { Thickness(mm) : (KA180)0.21; (K180)0.24; } \\
\text { (KLB175)0.22; (S120)0.19 } \\
\text { Ring crush(kgf) : (KA180)21.0; (K180)20.2; } \\
\text { (KLB175)25.0; (S120)8.4 }\end{array}$} \\
\hline $\mathrm{B} / \mathrm{F}$ & $\varpi$ & KA180/K180/K180 & \\
\hline $\mathrm{AB} / \mathrm{F}$ & $\approx$ & KLB175/K180/K180/K180/KLB175 & \\
\hline $\mathrm{BB} / \mathrm{F}$ & $\approx z$ & KA180/K180/S1205/K180/K180 & \\
\hline
\end{tabular}

1) (DW) OL/OC/ML/IC/IL; (SW) OL/C/IL (OL=outer liner, $\mathrm{ML}=$ middle liner, IL=inner liner, OC=outer corrugating medium, $\mathrm{IC}=$ inner corrugating medium, $\mathrm{C}=$ corrugating medium)

2)-5) KA; outer liner contained UKP (25\%) + AOCC (10\%) + KOCC (65\%), KLB; outer liner contained UKP (40\%) + AOCC (30\%) + KOCC (30\%), K and S; KOCC (100\%), UKP=unbleached kraft pulp, $\mathrm{AOCC}=$ American old corrugated container, $\mathrm{KOCC}=$ Korean old corrugated container

\section{Methods}

A sinusoidal sweep vibration test was performed to analyze the vibration transmissibility of corrugated paperboard for corrugation shape and equilibrium atmospheric conditions. In the test, vibration acceleration was $0.5 \mathrm{G}$ (zero to peak), sweep rate was 1 octave/min. and sweep mode was logarithmic rate based on the ASTM D3580.1

Generally, excitation frequencies were determined by considering the frequency range during transportation and testing time. In study, excitation frequencies were applied step by step from $5 \mathrm{~Hz}$ to $400 \sim 700 \mathrm{~Hz}$ and these were decided by dividing $0.80 \sim 5.64 \mathrm{kPa}$ of static stress range with 3 levels in consideration of control range of vibration table.

Corrugated paperboard specimen was positioned by fastening with two horizontal screws and pre-loaded with $14.72 \mathrm{~N}$ so that specimen experience same conditions with transportation condition during vibration test. Corrugated paperboard specimen was conditioned in a constant temperature and humidity chamber for 36 hours and laboratory was preserved with $23^{\circ} \mathrm{C}-\mathrm{rh} 50 \%$.

\section{RESULTS AND DISCUSSION}

\section{Vibration transmissibility for equilibrium atmos- pheric conditions}

Any cushioning material can be represented by transmissibility-frequency curve that is expressed as a nondimensional transmissibility that is ratio of its response amplitude to the excitation amplitude. As indicated in Fig. 4, the shape of the curve has three regions which are non-amplified region, amplified region and isolation region affected by the degree of damping in the system. Resonance exists at the frequency where transmissibility is maximized and the resonance is at least one for the cushioning materials. Because of the non-uniformity of corrugated paperboard, the transmissibility-frequency curve of it has more complicated shapes than uniform materials and the transmissibility at first resonance was far greater than transmissibility at other resonance. The lowest resonant frequency should be significantly considered for cushioning materials in packaging design.
Accordingly, this study analyzed only first resonance that exists in the low frequency region.

Table 2 shows the resonant frequency and maximum transmissibility of corrugated paperboard for each static stress according to the equilibrium atmospheric conditions of corrugated paperboard for the corrugation shapes. Even though resonant frequency was decreased as static stress was increased, maximum transmissibility did not show evident trends. Result of resonant frequency that decreased with increasing static stress follows the natural frequency's expression $f_{n}=(1 / 2 \pi) \sqrt{\mathrm{k} / \mathrm{m}}$ ( $m=$ mass, $k=$ spring stiffness coefficient) while considering the cushioning material include block as singledegree-of-freedom and represents the linearity of corrugated paperboard. The more resonant frequency was increased, the wider amplifying region of vibration became. These results correspond with the vibration features of multi-stacked corrugated paperboard boxes which have been presented by Godshall (1971). ${ }^{6}$

Within an applied static stress range, DW corrugated paperboard shows a small resonant frequency than that of SW corrugated paperboard but has high transmissibility. In the case of DW corrugated paperboard, it was proven that complex behavior of two corrugations for external vibration brings about the amplification of vibration. For corrugation shapes, $\mathrm{B} / \mathrm{F}$ was ranked first in highest resonant frequency ahead of $\mathrm{A} / \mathrm{F}, \mathrm{BB} / \mathrm{F}, \mathrm{AB} / \mathrm{F}$. BB/F was ranked first in maximum transmissibility ahead of $\mathrm{AB} / \mathrm{F}, \mathrm{A} / \mathrm{F}, \mathrm{B} / \mathrm{F}$

Figs. 4 and 5 show that resonant frequency and maximum transmissibility of corrugated paperboard were decreased when relative humidity was increased for all kinds of corrugated paperboard specimens and applied static stress. The reason for this is that corrugated paperboard stiffness was decreased as corrugated paperboard absorbed the humidity with increasing relative humidity. The decreasing rate of maximum transmissibility with increasing relative humidity is higher for SW corrugated paperboard than DW corrugated paperboard because the moisture absorption mechanism of SW corrugated paperboard is more sensitive than that of DW corrugated paperboard. 
Table 2. Maximum vibration transmissibility ( $T r)$ and resonant frequency (fr) for each combination of flute, static stress $\left(\sigma_{\mathrm{s}}\right)$ and environmental conditions

\begin{tabular}{|c|c|c|c|c|c|c|c|c|c|}
\hline \multirow{2}{*}{$\begin{array}{l}\text { Environmental } \\
\text { conditions }\end{array}$} & \multirow{2}{*}{$\begin{array}{c}\sigma \mathrm{S} \\
(\mathrm{kPa})\end{array}$} & \multicolumn{2}{|l|}{$\mathrm{AB} / \mathrm{F}$} & \multicolumn{2}{|l|}{$\mathrm{BB} / \mathrm{F}$} & \multicolumn{2}{|l|}{$\mathrm{A} / \mathrm{F}$} & \multicolumn{2}{|l|}{$\mathrm{B} / \mathrm{F}$} \\
\hline & & fr $(\mathrm{Hz})$ & $\operatorname{Tr}$ & fr $(\mathrm{Hz})$ & $\operatorname{Tr}$ & fr $(\mathrm{Hz})$ & $\operatorname{Tr}$ & fr $(\mathrm{Hz})$ & $\operatorname{Tr}$ \\
\hline \multirow{5}{*}{$23^{\circ} \mathrm{C}-\mathrm{rh} 50 \%$} & 0.80 & 433 (124 589) & 5.5 & 409 (121 579) & 6.5 & 510 (221 ) & 4.3 & 534 (252 ) & 3.9 \\
\hline & 2.01 & $300(101 \sim 440)$ & 5.8 & $270(84 \sim 400)$ & 6.3 & $380(154 \sim 560)$ & 4.8 & 398 (174 602) & 4.5 \\
\hline & 3.22 & $210(85 \sim 330)$ & 6.8 & $183(78 \sim 290)$ & 7.5 & $284(85 \sim 403)$ & 5.8 & 303 (104 445) & 5.4 \\
\hline & 4.44 & $177(77 \sim 300)$ & 7.1 & $153(71 \sim 253)$ & 7.4 & 237 (83 359) & 6.0 & 260 (94 397) & 5.5 \\
\hline & 5.64 & $174(73 \sim 274)$ & 6.5 & $151(54 \sim 234)$ & 7.0 & 210 (81 339) & 6.2 & $240(87 \sim 372)$ & 5.7 \\
\hline \multirow{5}{*}{$23^{\circ} \mathrm{C}-\mathrm{rh} 70 \%$} & 0.80 & $375(148 \sim 550)$ & 5.2 & $351(152 \sim 525)$ & 5.8 & $472(192 \sim 691)$ & 3.4 & 481 (230 ) & 3.1 \\
\hline & 2.01 & $252(114 \sim 413)$ & 4.7 & 242 (110 388) & 5.5 & $360(144 \sim 545)$ & 3.7 & $376(157 \sim 561)$ & 3.2 \\
\hline & 3.22 & 175 (75 283) & 5.8 & $170(73 \sim 280)$ & 6.5 & $272(89 \sim 405)$ & 4.6 & $290(86 \sim 407)$ & 3.8 \\
\hline & 4.44 & $149(65 \sim 255)$ & 5.5 & $141(63 \sim 232)$ & 6.1 & $228(80 \sim 339)$ & 4.9 & 251 (93 378) & 4.1 \\
\hline & 5.64 & 147 (64 237 & 5.0 & 137 (54 220) & 5.7 & $201(79 \sim 313)$ & 5.2 & $236(95 \sim 360)$ & 4.4 \\
\hline \multirow{5}{*}{$23{ }^{\circ} \mathrm{C}-\mathrm{rh} 90 \%$} & 0.80 & $294(104 \sim 445)$ & 3.0 & $330(97 \sim 465)$ & 3.5 & $453(127 \sim 620)$ & 2.4 & $437(121 \sim 590)$ & 2.1 \\
\hline & 2.01 & 202 (80 333) & 4.2 & $220(81 \sim 344)$ & 4.9 & 331 (110 482) & 2.5 & $340(110 \sim 490)$ & 2.0 \\
\hline & 3.22 & $138(56 \sim 224)$ & 4.1 & $150(61 \sim 253)$ & 4.5 & $252(85 \sim 376)$ & 3.0 & $273(89 \sim 405)$ & 2.5 \\
\hline & 4.44 & $118(58 \sim 206)$ & 3.2 & $122(60 \sim 211)$ & 3.6 & 212 (73 319) & 3.1 & $241(90 \sim 377)$ & 3.4 \\
\hline & 5.64 & $116(50 \sim 197)$ & 2.8 & $121(52 \sim 203)$ & 3.3 & $189(79 \sim 301)$ & 2.7 & $223(89 \sim 347)$ & 3.1 \\
\hline
\end{tabular}

Note: 1. ( ) : frequency in amplified region

2. All values are the mean of three replicates.

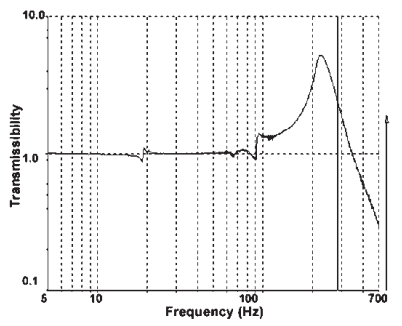

(a) $23^{\circ} \mathrm{C}$-rh $50 \%$

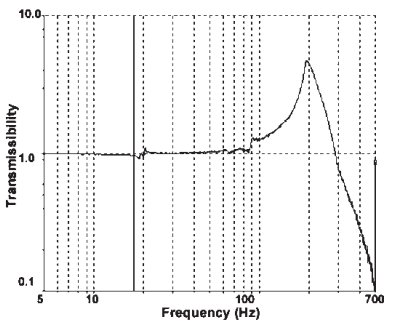

(b) $23^{\circ} \mathrm{C}-$ rh $70 \%$

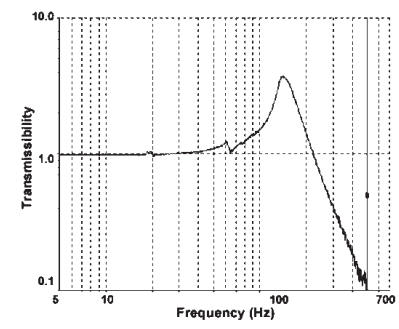

(c) $23^{\circ} \mathrm{C}$-rh $90 \%$

Fig. 4. Vibration transmissibility curves in varying environmental conditions.

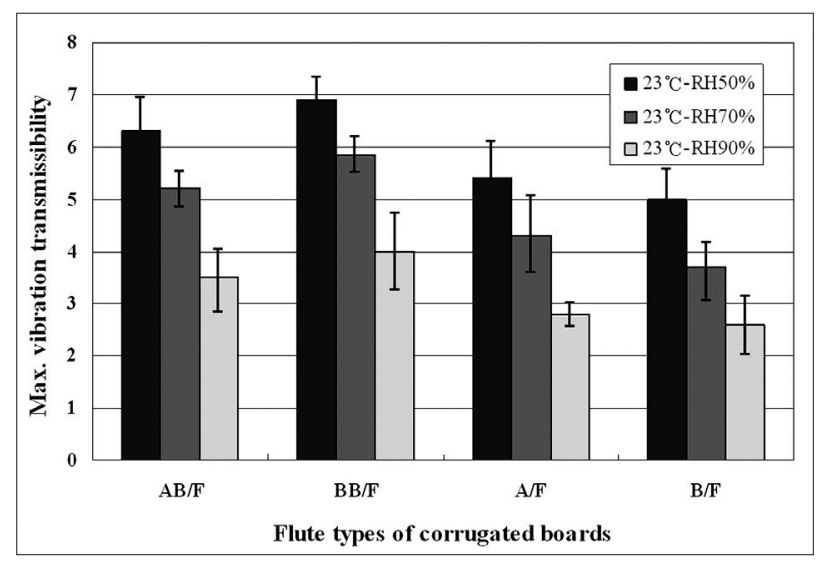

Fig. 5. Maximum vibration transmissibility in varying environmental conditions.

Resonant frequency of corrugated paperboard was usually more than $100 \mathrm{~Hz}$, which is higher than that of any other foamed plastic cushioning materials. Because stiffer cushioning materials have amplification-attenua- tion plot with quite high frequencies, no-amplification one to one region that transmits the input vibration without amplifying exists widely in the transmissibility-frequency curve. Accordingly, this stiffer cushioning material works well in the resonant frequency region while preserving its vibration amplitude. However, it can not attenuate the input vibration as it did in the isolation region.

\section{Prediction of damping ratio and relationship with equilibrium atmospheric conditions}

The damping ratio of a cushioning material is defined by the ratio of the real damping constant to the critical damping constant and will greatly affect the vibration transmissibility. In this study, the damping ratio was approximated by both resonance frequency and maximum vibration transmissibility.

The cushioning material including block system shown in Fig. 1 can be approximated by a linear vibration model in order to apply the linear vibration theory shown in Fig. 6. 


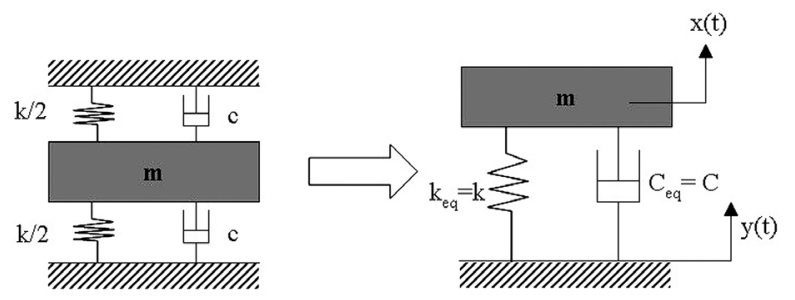

Fig. 6. Linear vibration model of single-degree-of-freedom.

Equation of vibration was induced by Newton's rule as equation (1) where $\mathrm{m}$ is mass of block, $y(t)=B \sin (\omega t)$ is displacement of excitation base.

$$
m \ddot{x}(t)=-c(\dot{x}-\dot{y})-k(x-y)
$$

Steady-state response for differential equation (1) is expressed by equation (2).

$$
\begin{aligned}
& x=X \sin (\omega t-\phi) \\
& X=B\left\{\frac{1+(2 \zeta \lambda)^{2}}{\left(1-\lambda^{2}\right)^{2}+(2 \zeta \lambda)^{2}}\right\}^{1 / 2}, \\
& \phi=\arctan \left\{\frac{(2 \zeta \lambda)^{3}}{1-\lambda^{2}+(2 \zeta \lambda)^{2}}\right\}
\end{aligned}
$$

Where $k$ is the stiffness coefficient of cushioning material, $c$ is viscous damping coefficient of cushioning material, $\omega$ is the excitation frequency of system, $\omega_{n}$ is natural frequency of system $(=\sqrt{\mathrm{k} / \mathrm{m}}), \lambda$ is the frequency ratio $\left(=\omega / \omega_{n}\right)$, and $\zeta$ is the damping ratio $\left(=c / 2 m \omega_{n}\right)$.

Displacement transmissibility ( $\mathrm{Tr}$ ) can be defined with the ratio of response amplitude to input amplitude by equation (4) and it is dimensionless equal to the transmissibility of force and accelerations.

$$
T_{r}=\frac{X}{B}=\left\{\frac{1+(2 \zeta \lambda)^{2}}{\left(1-\lambda^{2}\right)^{2}+(2 \zeta \lambda)^{2}}\right\}^{1 / 2}
$$

Equation (4) shows that transmissibility of vibration is greatly related with both frequency and damping ratio. In the case of little damping, damping ratio can be approximated by equation (5) because resonance usually happens around the value $\lambda \doteqdot 1$.

$$
\zeta=\frac{1}{2}\left(\frac{1}{T_{r}^{2}-1}\right)^{1 / 2}
$$

Table 3 shows the results of the approximated damping ratio according to both corrugation shape and equilibrium atmospheric conditions which are based on experimental data shown in Table 2 using equation (5). For the damping ratio according to corrugation shapes, $\mathrm{B} / \mathrm{F}$ was ranked first ahead of $\mathrm{A} / \mathrm{F}, \mathrm{BB} / \mathrm{F}$, and $\mathrm{AB} / \mathrm{F}$. It was shown that the damping ratio of SW corrugated paperboards was higher than that of DW corrugated paperboards. It also has increased with increasing relative humidity. And SW corrugated paperboard shows a higher increasing rate of damping ratio than that of DW corrugated paperboard. These results show that there is a different mechanism in moisture absorption between SW corrugated board and DW corrugated paperboard.

Table 3 also shows the maximum dynamic stress at resonance calculated by experimental data shown in

Table 3. Calculated damping ratio $(\zeta)$ and maximum dynamic stress $\left(\sigma_{\mathrm{d}}\right)$ for each combination of flute, static stress $\left(\sigma_{\mathrm{s}}\right)$ and environmental conditions

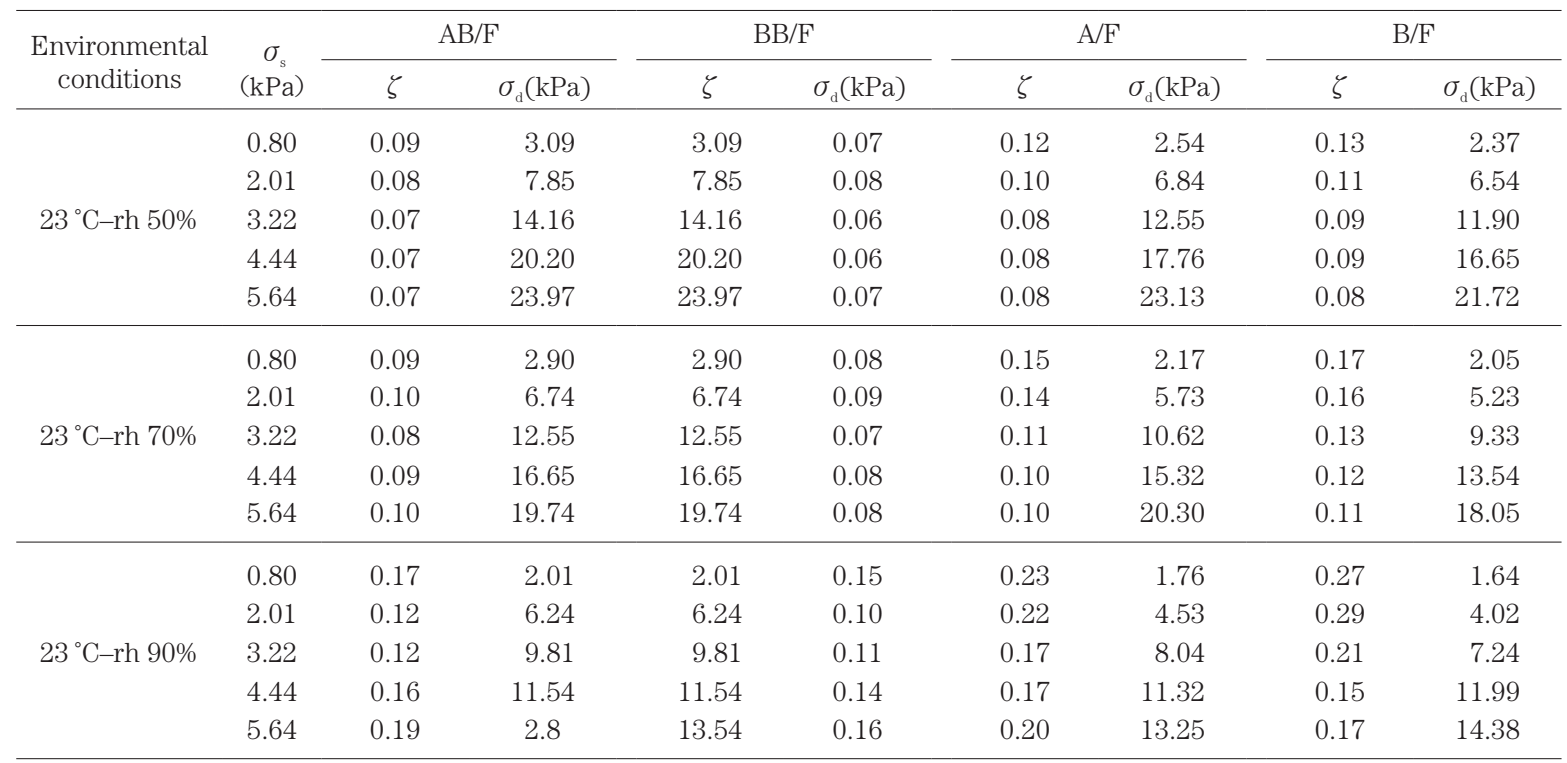

Notes :

1. maximum dynamic stress, $\sigma_{\mathrm{d}}=\sigma_{\mathrm{s}}\left(1+\mathrm{G}_{\mathrm{i}} \mathrm{T}_{\mathrm{r}}\right.$ ) (where, $\sigma_{\mathrm{s}}$ is static stress, $\mathrm{G}_{\mathrm{i}}$ is input acceleration level G's, and $\mathrm{T}_{\mathrm{r}}$ is maximum vibration transmissibility)

2. All values are the mean of three replicates. 


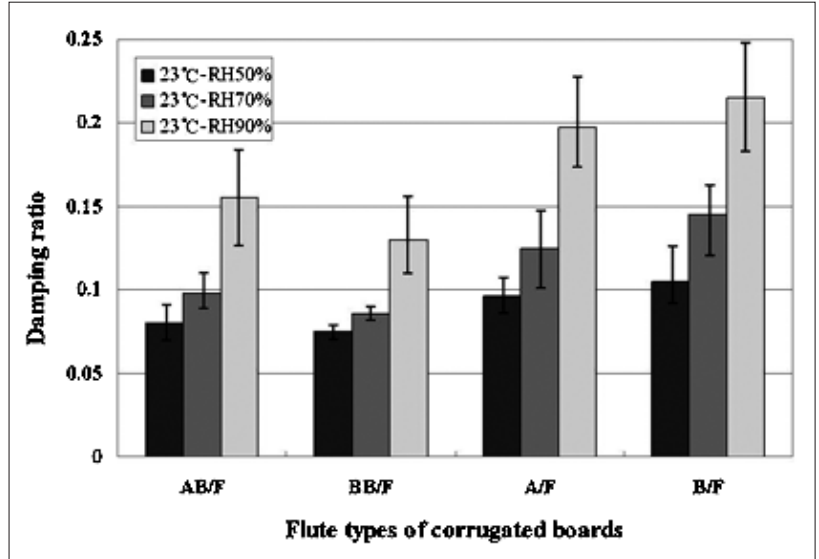

Fig. 7. Calculated damping ratio in varying environmental conditions.

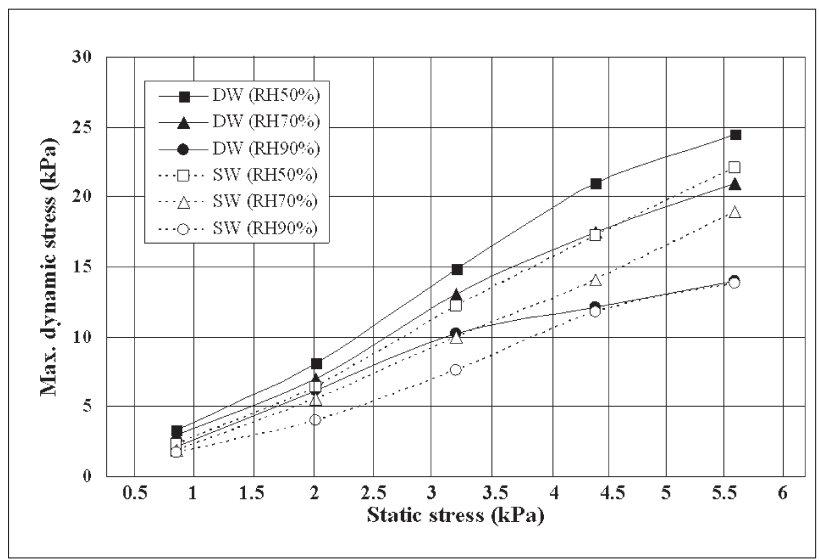

Fig. 8. Calculated maximum dynamic stress in varying environmental conditions.

Table 2 using Newton's rule. The maximum dynamic stress is considered as the impulsive maximum stress per unit area due to static stress including the dynamic stress from vibration. Repeated maximum dynamic stress could lead to fatigue damage for packaged products.

The maximum dynamic stress of all corrugated paperboard was affected by static stress and it decreased with increasing relative humidity. For the maximum dynamic stress of corrugated paperboard according to corrugation shapes, $\mathrm{BB} / \mathrm{F}$ was ranked first ahead of $\mathrm{AB} / \mathrm{F}$, $\mathrm{A} / \mathrm{F}$, and $\mathrm{B} / \mathrm{F}$ until the middle level of applied static stress. The maximum dynamic stress of DW corrugated board was higher than that of SW corrugated paperboard until the middle level of applied static stress. There was not a significant difference in maximum dynamic stress at higher static stress levels.

Characteristics of cushioning materials required for protecting products from impact and vibration during transportation are different. Namely, damping ratio is needed for the cushioning materials in vibrating conditions but increasing damping ratio can diminish the elasticity of cushioning materials and may result in impact damage on products. Accordingly, cushioning material should be optimized for its applications and products by considering both the vibration with transmissibility-fre- quency curve and the impact with cushioning curve.

\section{SUMMARY AND CONCLUSIONS}

Because of merits for environmental preservation, a paper-based cushioning material has been widely used and its applications have been expanded. In the case of corrugated paperboard, applications for cushioning materials were greatly increased beyond their conventional use in packaging containers.

In order to optimize the application of corrugated paperboard for packaging cushioning materials, it is essential that both a vibration transmissibility test and drop shock test be completed for the transmissibility-frequency curve and dynamic cushion curve respectively.

Accordingly, this study measured resonant frequency and maximum vibration transmissibility for a corrugated paperboard according to both corrugation shape and the equilibrium atmospheric conditions by a sinusoidal sweep vibration test. Additionally, damping ratio and maximum dynamic stress were evaluated by linear vibration theory.

The following are conclusions and summaries.

(1) Resonant frequency was linearly decreased with increasing static stress, whereas maximum vibration transmissibility has insignificant trends with increasing static stress.

(2) Resonant frequency of SW corrugated paperboard was higher than that of DW corrugated paperboard, however a maximum vibration transmissibility of DW corrugated paperboard was higher than that of SW corrugated paperboard in the range $0.80 \sim 5.64 \mathrm{kPa}$ of static stress. These results indicate that a complex mechanism of the upper and lower corrugation in DW corrugated paperboard affects the amplification of excitation vibration.

(3) Both resonant frequency and maximum vibration transmissibility were decreased with increasing relative humidity. The decreasing rate of maximum vibration transmissibility in SW corrugated paperboard was higher than that of DW. So, it can be concluded that the difference of corrugation shape and moisture absorption mechanism are important reasons for the resonant frequency and maximum vibration transmissibility of DW and SW.

(4) The damping ratio evaluated in SW corrugated paperboard was generally higher than that of DW corrugated paperboard and it was greatly increased by relative humidity conditions. Additionally, the increasing rate of damping ratio was higher in SW corrugated paperboard than in DW corrugated paperboard. A maximum dynamic stress was significantly affected by static stress while decreased with increasing relative humidity.

\section{REFERENCES}

ASTM D3580. Standard test methods for vibration (vertical linear motion) test of products 
Biancolini ME and C. Brutti 2003 Numerical and experimental investigation of the strength of corrugated board packages. Packaging Technology and Science, $\mathbf{1 6}(2)$ : 47-60

Bronkhorst CA and J. R. Riedemann 1994 The creep deformation behavior of corrugated containers in a cycle moisture environment. Proceedings of the Symposium on moisture creep behavior of paper and board, Stockholm, Sweden, 249-273

Guo Y and J. Zhang 2004 Shock absorbing characteristics and vibration transmissibility of honeycomb paperboard. Shock and Vibration, 11(5-6): 521-531

Guo Y, Y. Fu and W. Zhang 2008 Creep properties and recoverability of double-wall corrugated paperboard. Experimental Mechanics, 48(3): 327-333

Godshall W. D. 1971 Frequency response, damping, and transmissibility characteristics of top-loaded corrugated container. United States Department of Agriculture, Forest Service, Forest Products Laboratory, Research Paper FPL 160

Harris C. M. and A. G. Piersol 2002 Shock and vibration handbook, 5th Edition New York: McGraw-Hill

Hahn E. K., A. D. Rudo, B. S. Westerlind and L. A. Carisson 1992
Compressive strength of edge-loaded corrugated board panels. Exp. Mech., 32(3): 259-265

Lee M. H. and J. M. Park 2004 Flexural stiffness of selected corrugated structures. Packaging Technology and Science, 17(5): $275-286$

McKee R. C., J. W. Gander and J. R. Wachuta 1963 Compressive strength formula for corrugated fiberboard boxes. Paperboard Packaging, 48(8): 149-159

MIL-HDBK-304C, Department of Defense Handbook: Package cushioning design. 1997; Department of Defense in United States of America

Sek M and J. Kirkpatrick 1997 Prediction of the cushioning properties of corrugated fiberboard from static and quasi-dynamic compression data. Packaging Technology and Science, 10 (2): $87-94$

Urbanik T. J. 2001 Effect of corrugated flute shape on fiberboard edgewise crush strength and bending stiffness. J. Pulp Pap Sci., 27(10): 330-335

Wang D. M. and Z. W. Wang 2008 Experimental investigation into the cushioning properties of honeycomb paperboard. Packaging Technology and Science, 21(6): 309-316 